\title{
Deciding Termination of Query Evaluation in Transitive-Closure Logics for Constraint Databases
}

\author{
Floris Geerts ${ }^{\star 1}$ and Bart Kuijpers ${ }^{2}$ \\ 1 University of Helsinki \\ Helsinki Institute for Information Technology \\ PO Box 26 (Teollisuuskatu 23), Fin-00014, Helsinki, Finland \\ floris.geerts@cs.helsinki.fi \\ 2 University of Limburg \\ Dept. of Mathematics, Physics and Computer Science \\ Universitaire Campus, 3590 Diepenbeek, Belgium \\ bart.kuijpers@luc.ac.be
}

\begin{abstract}
We study extensions of first-order logic over the reals with different types of transitive-closure operators as query languages for constraint databases that can be described by Boolean combinations of polynomial inequalities over the reals. We are in particular interested in deciding the termination of the evaluation of queries expressible in these transitive-closure logics. It turns out that termination is undecidable in general. However, we show that the termination of the transitive closure of a continuous function graph in the two-dimensional plane, viewed as a binary relation over the reals, is decidable, and even expressible in first-order logic over the reals. Based on this result, we identify a particular transitive-closure logic for which termination of query evaluation is decidable and which is more expressive than first-order logic over the reals. Furthermore, we can define a guarded fragment in which exactly the terminating queries of this language are expressible.
\end{abstract}

\section{Introduction}

The framework of constraint databases, introduced in 1990 by Kanellakis, Kuper and Revesz [12] and by now well-studied [17], provides an elegant and powerful model for applications that deal with infinite sets of points in some real space $\mathbb{R}^{n}$, for instance spatial databases. In the setting of the constraint model, these infinite sets are finitely represented as Boolean combinations of polynomial equalities and inequalities over the reals. For example, the spatial database consisting of the set of points on the northern hemisphere together with the points on the equator of the unit sphere in the three-dimensional space $\mathbb{R}^{3}$ can be represented by the formula $x^{2}+y^{2}+z^{2}=1 \wedge z \geq 0$. The relational calculus augmented with polynomial constraints, FO for short, is the

* The research presented here was done while this author was at the University of Limburg. 
standard first-order query language for constraint databases. The FO-sentence $(\exists r)(\forall x)(\forall y)(\forall z)\left(S(x, y, z) \rightarrow x^{2}+y^{2}+z^{2}<r^{2}\right)$ expresses that the threedimensional spatial relation $S$ is bounded.

Although many interesting properties can be expressed in FO, its most important deficiency is that its expressive power is rather limited. For instance, several practically relevant topological properties of spatial data, such as connectivity and reachability, are not expressible in FO and various people have proposed and studied extensions of FO with tractable recursion mechanisms to obtain more expressive languages $[2,11,14-16]$. In analogy with the classical graph connectivity query, which cannot be expressed in the standard relational calculus but which can be expressed in the relational calculus augmented with a transitive-closure operator, also extensions of FO with various transitive-closure operators have been proposed to obtain languages that are more expressive, in particular that allow the expression of connectivity and reachability queries and that are even computationally complete. Recently, the present authors introduced FO+TC and FO+TCS, two languages in which an operator is added to FO that allows the computation of the transitive closure of unparameterized sets in some $\mathbb{R}^{2 k}[10]$. In the latter language also FO-definable stop conditions are allowed to control the evaluation of the transitive-closure. Later on, Kreutzer has studied the language that we refer to as FO+KTC [15], which is an extension of FO with a transitive-closure operator that may be applied to parameterized sets and in which the evaluation of a transitive-closure expression may be controlled by the termination of particular paths in its computation rather than by the termination of the transitive closure of the complete set. The fragments of $\mathrm{FO}+\mathrm{TCS}$ and $\mathrm{FO}+\mathrm{KTC}$, that does not use multiplication, are shown to be computationally complete on databases definable by linear constraints $[10,15]$.

In all of these transitive-closure languages, we face the well-know fact that recursion involving arithmetic over an infinite domain, such as the reals with addition and multiplication in this setting, is not guaranteed to terminate. In this paper, we are interested in termination of query evaluation in these different transitive-closure logics and in particular in deciding termination. We show that the termination of the evaluation of a given query, expressed in any of these languages, on a given input database is undecidable as soon as the transitive closure of 4-ary relations is allowed. In fact, a known undecidable problem in dynamical systems theory, namely deciding nilpotency of functions from $\mathbb{R}^{2}$ to $\mathbb{R}^{2}[3,4]$, can be reduced to our decision problem. When the transitive-closure operator is restricted to work on binary relations, the matter is more complicated. We show the undecidability of termination for FO+TCS restricted to binary relations. However, both for $\mathrm{FO}+\mathrm{TC}$ and $\mathrm{FO}+\mathrm{KTC}$ restricted to binary relations, finding an algorithm for deciding termination would also solve some outstanding open problems in dynamical systems theory. Indeed, a decision procedure for $\mathrm{FO}+\mathrm{TC}$ restricted to binary relations would solve the nilpotency problem for functions from $\mathbb{R}$ to $\mathbb{R}$ and a decision procedure for $\mathrm{FO}+\mathrm{KTC}$ restricted to binary relations would solve the point-to-fixed-point problem. Both these problems are already open for some time $[3,13]$. 

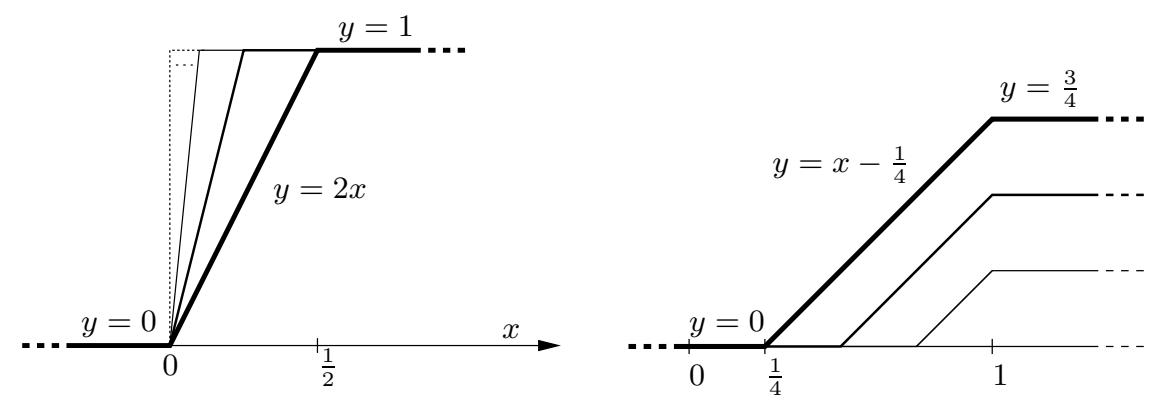

Fig. 1. On the left, a function graph (thick) with non-terminating transitive closure (thin). On the right, a function graph (thick) with terminating transitive closure (thin).

For $\mathrm{FO}+\mathrm{TC}$ restricted to binary relations, we have obtained a positive decidability result, however. A basic problem in this context is deciding whether the transitive closure of a fixed subset of the two-dimensional plane, viewed as a binary relation over the reals, terminates. Even if these subsets are restricted to be the graphs of possibly discontinuous functions from $\mathbb{R}$ to $\mathbb{R}$, this problem is already puzzling dynamical system theorists for a number of years (it relates to the above mentioned point-to-fixed-point problem). However, when we restrict our attention to the transitive closure of continuous function graphs, we can show that the termination of the transitive closure of these figures is decidable. As an illustration of possible inputs for this decision problem, two continuous function graphs are given in Fig. 1. The one on the left has a non-terminating transitive closure, but the one on the right terminates after four iterations. Furthermore, we show that this decision procedure is expressible in FO. In the course of our proof, we also give a stronger version of Sharkovski1's theorem [1] from dynamical systems theory for terminating continuous functions. We also extend another result in this area, namely, we show that nilpotency of continuous semi-algebraic functions is decidable and that this decision procedure is even expressible in FO. Previously, this result was only stated, without proof, for continuous piecewise affine functions [4].

Based on this decision result, we define a fragment of $\mathrm{FO}+\mathrm{TC}$ in which the transitive-closure operator is restricted to work on graphs of continuous functions from $\mathbb{R}$ to $\mathbb{R}$. Termination of queries in this language is shown to be decidable. Furthermore, we define a guarded fragment of this transitive-closure logic in which only, and all, terminating queries can be formulated. We also show that this very restricted form of transitive closure yields a language that is strictly more expressive than FO.

This paper is organized as follows. In Section 2, we define constraint databases, the query language FO and several extensions with transitive-closure operators. In Section 3, we give general undecidability results. In Section 4, we give a procedure to decide termination of the transitive closure of continuous function graphs in the plane. In Section 5, we study the extension of FO with a transitive closure operator that is restricted to work on continuous function 
graphs. In this section, we also describe a guarded fragment of this language and give expressiveness results. The paper concludes with some remarks.

\section{Definitions and Preliminaries}

In this section, we define constraint databases and their standard first-order query language FO. We also define existing extensions of this logic with different transitive-closure operators: FO+TC, FO+TCS and FO+KTC.

\subsection{Constraint Databases and First-Order Logic over the Reals}

Let $\mathbb{R}$ denote the set of the real numbers, and $\mathbb{R}^{n}$ the $n$-dimensional real space (for $n \geq 1$ ).

Definition 1. An $n$-dimensional constraint database ${ }^{3}$ is a geometrical figure in $\mathbb{R}^{n}$ that can be defined as a Boolean combination (union, intersection and complement) of sets of the form $\left\{\left(x_{1}, \ldots, x_{n}\right) \mid p\left(x_{1}, \ldots, x_{n}\right)>0\right\}$, where $p\left(x_{1}, \ldots, x_{n}\right)$ is a polynomial with integer coefficients in the real variables $x_{1}, \ldots$, $x_{n}$.

We remark that in mathematical terminology, constraint databases are called semi-algebraic sets [5]. If a constraint database can be described by linear polynomials only, we refer to it as a linear constraint database.

In this paper, we will use FO, the relational calculus augmented with polynomial inequalities as a basic query language.

Definition 2. A formula in FO, over an $n$-dimensional input database, is a firstorder logic formula, $\varphi\left(y_{1}, \ldots, y_{m}, S\right)$, built, using the logical connectives and quantification over real variables, from two kinds of atomic formulas, namely $S\left(x_{1}, \ldots, x_{n}\right)$ and $p\left(x_{1}, \ldots, x_{k}\right)>0$, where $S$ is a $n$-ary relation name representing the input database and $p\left(x_{1}, \ldots, x_{k}\right)$ is a polynomial with integer coefficients in the real variables $x_{1}, \ldots, x_{k}$.

In the expression $\varphi\left(y_{1}, \ldots, y_{m}, S\right), y_{1}, \ldots, y_{m}$ denote the free variables. Variables in such formulas are assumed to range over $\mathbb{R}$. Tarski's quantifier-elimination procedure for first-order logic over the reals guarantees that FO expressions can be evaluated effectively on constraint database inputs and their result is a constraint database (in $\mathbb{R}^{m}$ ) that also can be described by means of polynomial constraints over the reals $[6,21]$.

If $\varphi\left(y_{1}, \ldots, y_{m}, S\right)$ is an FO formula, $a_{1}, \ldots, a_{m}$ are reals, and $A$ is an $n$ dimensional constraint database, then we denote by $\left(a_{1}, \ldots, a_{m}, A\right) \models \varphi\left(y_{1}, \ldots\right.$, $\left.y_{m}, S\right)$ that $\left(a_{1}, \ldots, a_{m}, A\right)$ satisfies $\varphi$. We denote by $\varphi(A)$ the set $\left\{\left(a_{1}, \ldots, a_{m}\right) \mid\right.$ $\left.\left(a_{1}, \ldots, a_{m}, A\right) \models \varphi\left(y_{1}, \ldots, y_{m}, S\right)\right\}$.

The fragment of FO in which multiplication is disallowed is called FOLin. This fragment is closed on the class of linear constraint databases [17].

\footnotetext{
${ }^{3}$ Spatial databases in the constraint model are usually defined as finite collections of such geometrical figures. We have chosen the simpler definition of a database as a single geometrical figure, but all results carry over to the more general setting.
} 


\subsection{Transitive-Closure Logics}

We now define a number of extensions of $\mathrm{FO}$ (and of $\mathrm{FO}_{\mathrm{Lin}}$ ) with different types of transitive-closure operators. Recently, we introduced and studied the first two extensions, $\mathrm{FO}+\mathrm{TC}$ and $\mathrm{FO}+\mathrm{TCS}[9,10]$. The latter extension, $\mathrm{FO}+\mathrm{KTC}$, is due to Kreutzer [15].

Definition 3. A formula in $\mathrm{FO}+\mathrm{TC}$ is a formula built in the same way as an FO formula, but with the following extra formation rule: if $\psi(\boldsymbol{x}, \boldsymbol{y})$ is a formula with $\boldsymbol{x}$ and $\boldsymbol{y} k$-tuples of real variables, and with all free variables of $\psi$ among $\boldsymbol{x}$ and $\boldsymbol{y}$ and if $\boldsymbol{s}, \boldsymbol{t}$ are $k$-tuples of real variables, then

$$
\left[\mathrm{TC}_{\boldsymbol{x} ; \boldsymbol{y}} \psi(\boldsymbol{x}, \boldsymbol{y})\right](\boldsymbol{s}, \boldsymbol{t})
$$

is also a formula which has as free variables those in $s$ and $\boldsymbol{t}$.

The semantics of a subformula of the above form (1) evaluated on a database $A$ is defined in the following operational manner: Start computing the following iterative sequence of $2 k$-ary relations: $X_{0}:=\psi(A)$ and $X_{i+1}:=X_{i} \cup\{(\boldsymbol{x}, \boldsymbol{y}) \in$ $\left.\mathbb{R}^{2 k} \mid(\exists \boldsymbol{z})\left(X_{i}(\boldsymbol{x}, \boldsymbol{z}) \wedge X_{0}(\boldsymbol{z}, \boldsymbol{y})\right)\right\}$ and stop as soon as $X_{i}=X_{i+1}$. The semantics of $\left[\mathrm{TC}_{\boldsymbol{x} ; \boldsymbol{y}} \psi(\boldsymbol{x}, \boldsymbol{y})\right](\boldsymbol{s}, \boldsymbol{t})$ is then defined as $(\boldsymbol{s}, \boldsymbol{t})$ belonging to the $2 k$-ary relation $X_{i}$.

Since every step in the above algorithm, including the test for $X_{i}=X_{i+1}$, is expressible in FO, every step is effective and the only reason why the evaluation may not be effective is that the computation does not terminate. In that case the semantics of the formula (1) (and any other formula in which it occurs as subformula) is undefined.

As an example of an $\mathrm{FO}+\mathrm{TC}$ formula over a 2-dimensional input database, we take $\left[\mathrm{TC}_{x ; y} S(x, y)\right](s, t)$. This expression, when applied to a 2-dimensional figure, returns the transitive closure of this figure, viewed as a binary relation over $\mathbb{R}$. For illustrations of the evaluation of this formula, we refer to the examples in Fig. 1 in the Introduction.

The language $\mathrm{FO}_{\mathrm{Lin}}+\mathrm{TC}$ consists of all $\mathrm{FO}+\mathrm{TC}$ formulas that do not use multiplication.

The following language, $\mathrm{FO}+\mathrm{TCS}$, is a modification of $\mathrm{FO}+\mathrm{TC}$ that incorporates a construction to specify explicit termination conditions on transitive closure computations.

Definition 4. A formula in FO+TCS is built in the same way as an FO formula, but with the following extra formation rule: if $\psi(\boldsymbol{x}, \boldsymbol{y})$ is a formula with $\boldsymbol{x}$ and $\boldsymbol{y} k$-tuples of real variables; $\sigma$ is an FO sentence over the input database and a special $2 k$-ary relation name $X$; and $s, t$ are $k$-tuples of real variables, then

$$
\left[\mathrm{TC}_{\boldsymbol{x} ; \boldsymbol{y}} \psi(\boldsymbol{x}, \boldsymbol{y}) \mid \sigma\right](\boldsymbol{s}, \boldsymbol{t})
$$

is also a formula which has as free variables those in $\boldsymbol{s}$ and $\boldsymbol{t}$. We call $\sigma$ the stop condition of this formula. 
The semantics of a subformula of the above form (2) evaluated on databases $A$ is defined in the same manner as in the case without stop condition, but now we stop not only in case an $i$ is found such that $X_{i}=X_{i+1}$, but also when an $i$ is found such that $\left(A, X_{i}\right) \models \sigma$, whichever case occurs first. As above, we also consider the restriction $\mathrm{FO}_{\mathrm{Lin}}+\mathrm{TCS}$. It was shown that $\mathrm{FO}_{\mathrm{Lin}}+\mathrm{TCS}$ is computationally complete, in the sense of Turing-complete on the polynomial constraint representation of databases (see Chap. 2 of [17]) on linear constraint databases [10].

Finally, we define FO+KTC. In finite model theory [8], transitive-closure logics, in general, allow the use of parameters. Also the language FO+KTC allows parameters in the transitive closure.

Definition 5. A formula in FO+KTC is a formula built in the same way as an FO formula, but with the following extra formation rule: if $\psi(\boldsymbol{x}, \boldsymbol{y}, \boldsymbol{u})$ is a formula with $\boldsymbol{x}$ and $\boldsymbol{y} k$-tuples of real variables, $\boldsymbol{u}$ some further $\ell$-tuple of free variables, and where $\boldsymbol{s}, \boldsymbol{t}$ are $k$-tuples of real terms, then

$$
\left[\mathrm{TC}_{\boldsymbol{x} ; \boldsymbol{y}} \psi(\boldsymbol{x}, \boldsymbol{y}, \boldsymbol{u})\right](\boldsymbol{s}, \boldsymbol{t})
$$

is also a formula which has as free variables those in $\boldsymbol{s}, \boldsymbol{t}$ and $\boldsymbol{u}$.

Since the free variables in $\psi(\boldsymbol{x}, \boldsymbol{y}, \boldsymbol{u})$ are those in $\boldsymbol{x}, \boldsymbol{y}$ and $\boldsymbol{u}$, here parameters are allowed in applications of the TC operator. The semantics of a subformula of the form (3), with $s=\left(s_{1}, \ldots, s_{k}\right)$, evaluated on a database $A$ is defined in the following operational manner: Let $I$ be the set of indices $i$ for which $s_{i}$ is a constant. Then we start computing the following iterative sequence of $(2 k+\ell)$ ary relations: $X_{0}:=\psi(A) \wedge \bigwedge_{i \in I}\left(s_{i}=x_{i}\right)$ and $X_{i+1}:=X_{i} \cup\left\{(\boldsymbol{x}, \boldsymbol{y}, \boldsymbol{u}) \in \mathbb{R}^{2 k+\ell}\right.$ $\left.(\exists \boldsymbol{z})\left(X_{i}(\boldsymbol{x}, \boldsymbol{z}, \boldsymbol{u}) \wedge \psi(\boldsymbol{z}, \boldsymbol{y}, \boldsymbol{u})\right)\right\}$ and stop as soon as $X_{i}=X_{i+1}$. The semantics of $\left[\mathrm{TC}_{\boldsymbol{x} ; \boldsymbol{y}} \psi(\boldsymbol{x}, \boldsymbol{y}, \boldsymbol{u})\right](\boldsymbol{s}, \boldsymbol{t})$ is then defined as $(\boldsymbol{s}, \boldsymbol{t}, \boldsymbol{u})$ belonging to the $(2 k+\ell)$-ary relation $X_{i}$.

We again also consider the fragment $\mathrm{FO}_{\text {Lin }}+\mathrm{KTC}$ of this language. It was shown that $\mathrm{FO}_{\mathrm{Lin}}+\mathrm{KTC}$ is computationally complete on linear constraint databases [15].

For all of the transitive-closure logics that we have introduced in this section, we consider fragments in which the transitive-closure operator is restricted to work on relations of arity at most $2 k$ and we denote this by adding $2 k$ as a superscript to the name of the language. For example, in the language FO+TCS ${ }^{4}$, the transitive closure is restricted to binary and 4-ary relations.

\section{Undecidability of the Termination of the Evaluation of Transitive-Closure Formulas}

The decision problems that we consider in this section and the next take couples $(\varphi, A)$ as input, where $\varphi$ is an expression in the transitive-closure logic under consideration and $A$ is an input database, and the answer to the decision problem 
is yes if the computation of the semantics of $\varphi$ on $A$ (as defined for that logic) terminates. We then say, for short, that $\varphi$ terminates on $A$.

We give the following general undecidability result concerning termination. In the proof and further on, the notion of nilpotency of a function will be used: a function $f: \mathbb{R}^{n} \rightarrow \mathbb{R}^{n}$ is called nilpotent if there exists a natural number $k \geq 1$ such that for all $\boldsymbol{x} \in \mathbb{R}^{n}, f^{k}(\boldsymbol{x})=(0, \ldots, 0)$.

Theorem 1. It is undecidable whether a given formula in $\mathrm{FO}+\mathrm{TC}^{4}$ terminates on a given input database.

Proof (sketch). We reduce deciding whether a piecewise affine function ${ }^{4} f$ : $\mathbb{R}^{2} \rightarrow \mathbb{R}^{2}$ is nilpotent to deciding whether the evaluation of a formula in $\mathrm{FO}+\mathrm{TC}^{4}$ terminates. So, assume that termination of formulas in $\mathrm{FO}+\mathrm{TC}^{4}$ is decidable. For a given piecewise affine function $f: \mathbb{R}^{2} \rightarrow \mathbb{R}^{2}$, graph $(f)$, the graph of $f$, is a semi-algebraic subset of $\mathbb{R}^{4}$. We give a procedure to decide whether $f$ is nilpotent:

Step 1. Decide whether the FO+TC ${ }^{4}$-query $\left[\mathrm{TC}_{x_{1}, x_{2} ; y_{1}, y_{2}} S\left(x_{1}, x_{2}, y_{1}, y_{2}\right)\right]\left(s_{1}, s_{2}\right.$, $\left.t_{1}, t_{2}\right)$ terminates on the input graph $(f)$; if the answer is no, then return no, else continue with Step 2.

Step 2. Compute $f^{1}\left(\mathbb{R}^{2}\right), f^{2}\left(\mathbb{R}^{2}\right), f^{3}\left(\mathbb{R}^{2}\right), \ldots$ and return yes if this ends with $\{(0,0)\}$, else return no.

This algorithm decides correctly whether $f$ is nilpotent, since for a nilpotent $f$, the evaluation of the transitive closure of $\operatorname{graph}(f)$ will terminate, and the process in Step 2 is therefore also guaranteed to terminate. Since nilpotency of piecewise affine functions from $\mathbb{R}^{2}$ to $\mathbb{R}^{2}$ is known to be undecidable [4], this completes the proof.

The following corollary follows from the previous theorem and the fact that $\mathrm{FO}+\mathrm{TC}^{4}$-formulas are in $\mathrm{FO}+\mathrm{KTC}^{4}$.

Corollary 1. It is undecidable whether a given formula in $\mathrm{FO}+\mathrm{KTC}^{4}$ terminates on a given input database.

For transitive-closure logics with stop-condition, we even have undecidability for transitive closure restricted to binary relations.

Theorem 2. It is undecidable whether a given formula in $\mathrm{FO}+\mathrm{TCS}^{2}$ terminates on a given input database.

ProOF (sketch). We prove this result by reducing the undecidability of a variant of Hilbert's 10th problem to it. This problem is deciding whether a polynomial $p\left(x_{1}, \ldots, x_{13}\right)$ in 13 real variables and with integer coefficients has a solution in

${ }^{4}$ A function $f: \mathbb{R}^{n} \rightarrow \mathbb{R}^{n}$ is called piecewise affine if its graph is a linear semialgebraic subset of $\mathbb{R}^{n} \times \mathbb{R}^{n}$. 
the natural numbers $[7,19]$. For any such polynomial $p\left(x_{1}, \ldots, x_{13}\right)$, let $\sigma_{p}$ be the FO-expressible stop-condition:

$$
\left(\exists x_{1}\right) \cdots\left(\exists x_{13}\right)\left(\bigwedge_{i=1}^{13} X\left(-1, x_{i}\right) \wedge p\left(x_{1}, \ldots, x_{13}\right)=0\right) .
$$

Since, in consecutive iterations of the computation of the transitive closure of the graph of $y=x+1,-1$ is mapped to $0,1,2, \ldots$, it is easy to see that $p\left(x_{1}, \ldots, x_{13}\right)$ has an integer solution if and only if $\left[\mathrm{TC}_{x ; y}(y=x+1) \mid \sigma_{p}\right](s, t)$ terminates. Since the above mentioned Diophantine decision problem is undecidable, this completes the proof.

The results in this section are complete for the languages $\mathrm{FO}+\mathrm{TC}, \mathrm{FO}+\mathrm{TCS}$ and $\mathrm{FO}+\mathrm{KTC}$, apart from the cases $\mathrm{FO}+\mathrm{TC}^{2}$ and $\mathrm{FO}+\mathrm{KTC}^{2}$. The former case will be studied in the next sections. For the latter case, we remark that an open problem in dynamical systems theory, namely, the point-to-fixed-point problem reduces to it. This open problem is the decision problem that asks whether for a given algebraic number $x_{0}$ and a given piecewise affine function $f: \mathbb{R} \rightarrow \mathbb{R}$, the sequence $x_{0}, f\left(x_{0}\right), f^{2}\left(x_{0}\right), f^{3}\left(x_{0}\right), \ldots$ reaches a fixed point. Even for piecewise linear functions with two non-constant pieces this problem is open [3,13]. It is clear that this point-to-fixed-point problem can be expressed in $\mathrm{FO}+\mathrm{KTC}^{2}$. So, we are left with the following unsolved problem.

Open problem 1. Is it decidable whether a given formula in $\mathrm{FO}+\mathrm{KTC}^{2}$ terminates on a given input database?

\section{Deciding Termination for Continuous Function Graphs in the Plane}

In this section, we study the termination of the transitive closure of a fixed semialgebraic subset of the plane, viewed as a binary relation over $\mathbb{R}$. We say that a subset $A$ of $\mathbb{R}^{2}$ has a terminating transitive closure, if the query expressed by $\left[\mathrm{TC}_{x ; y} S(x, y)\right](s, t)$ terminates on input $A$ using the semantics of FO+TC. In the previous section, we have shown that deciding nilpotency of functions can be reduced to deciding termination of the transitive closure of their function graphs. However, since it is not known whether nilpotency of (possible discontinuous) functions from $\mathbb{R}$ to $\mathbb{R}$ is decidable, we cannot use this reduction to obtain the undecidability in case of binary function graphs. We therefore have another unsolved problem:

Open problem 2. Is it decidable whether a given formula in $\mathrm{FO}+\mathrm{TC}^{2}$ terminates on a given input database?

There are obviously classes of functions for which deciding termination of their function graphs is trivial. An example is the class of the piecewise constant functions. In this section, we concentrate on a class that is non-trivial, namely 
the class of the continuous semi-algebraic ${ }^{5}$ functions from $\mathbb{R}$ to $\mathbb{R}$. The main purpose of this section is to prove the following theorem.

Theorem 3. There is a decision procedure that on input a continuous semialgebraic function $f: \mathbb{R} \rightarrow \mathbb{R}$ decides whether the transitive closure of $\operatorname{graph}(f)$ terminates. Furthermore, this decision procedure can be expressed by a formula in $\mathrm{FO}$ (over a 2-ary database that represents the graph of the input function).

Before we arrive at the proof of Theorem 3, we give a series of six technical lemma's. First, we introduce some terminology.

Let $f: \mathbb{R} \rightarrow \mathbb{R}$ be a continuous function and let $x$ be a real number. We call the set $\left\{f^{k}(x) \mid k \geq 0\right\}$ the orbit of $x$ (with respect to $f$ ). A real number $x$ is said to be a periodic point of $f$ if $f^{d}(x)=x$ for some natural number $d \geq 1$. And we call the smallest such $d$ the period of $x$ (with respect to $f$ ). Let $\operatorname{Per}(f)$ be the set of periodic points of $f$. If a real number $x$ is not a periodic point of $f$, but if $f^{k}(x)$ is periodic for some natural number $k \geq 1$, we call $x$ an eventually periodic point of $f$ and we call the smallest such number $k$ the run-up of $x$ (with respect to $f$ ). Finally, we call $f$ terminating if $\operatorname{graph}(f)$ has a terminating transitive closure.

The following lemma holds for arbitrary functions, not only for continuous ones. We also remark that Lemmas 1-4 hold for arbitrary functions, not only for semi-algebraic ones. We omit the proofs of Lemmas 1-4.

Lemma 1. The function $f: \mathbb{R} \rightarrow \mathbb{R}$ is terminating if and only if there exist natural numbers $k$ and $d$ such that for each $x \in \mathbb{R}, f^{k}(x)$ is a periodic point of $f$ of period at most d.

Lemma 2. Let $f: \mathbb{R} \rightarrow \mathbb{R}$ be a continuous function. If $f$ is terminating, then $\operatorname{Per}(f)$ is a non-empty, closed and connected part of $\mathbb{R}$. In particular, $\operatorname{Per}(f)=f^{k}(\mathbb{R})$ for some $k \geq 1$.

Lemma 3. Let $C$ be a non-empty, closed and connected part of $\mathbb{R}$. If $f: C \rightarrow C$ is a continuous function and if every $x \in C$ is periodic for $f$, then $f$ or $f^{2}$ is the identity mapping on $C$.

Lemma 4. For a continuous and terminating $f: \mathbb{R} \rightarrow \mathbb{R}, \operatorname{Per}(f)=\{x \in \mathbb{R} \mid$ $\left.f^{2}(x)=x\right\}$.

Denote by $C_{i}$ the set of fixed points of $f^{i}$, i.e., the set of $x \in \mathbb{R}$ for which $f^{i}(x)=x$. From the previous lemmas it follows that for continuous and terminating $f$,

$$
\operatorname{Per}(f)=C_{1} \cup C_{2},
$$

and that either $C_{2} \backslash C_{1}$ is empty and $C_{1}$ is non-empty or $C_{2} \backslash C_{1}$ is non-empty and $C_{1}$ is a singleton with the points of $C_{2} \backslash C_{1}$ appearing around $C_{1}$.

Sharkovskiî's theorem [1], one of the most fundamental result in dynamical system theory, tells us that for a continuous and terminating $f: \mathbb{R} \rightarrow \mathbb{R}$

\footnotetext{
${ }^{5}$ A function is called semi-algebraic if its graph is semi-algebraic.
} 

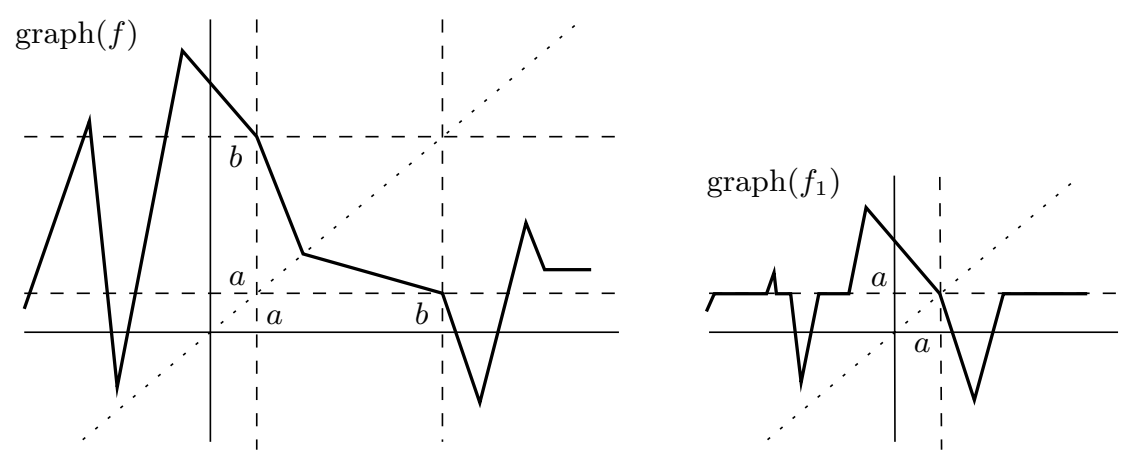

Fig. 2. Illustration of the construction of $f_{1}$ (right) from $f$ (left).

only periods $1,2,4, \ldots, 2^{d}$ can appear for some integer value $d$. The previous lemma has the following corollary which strengthens the result of Sharkovskilı's for terminating functions.

Corollary 2. If $f: \mathbb{R} \rightarrow \mathbb{R}$ is continuous and terminating, then $f$ can only have periodic points with periods 1 and 2.

Further on, in the proof of Theorem 3, for functions $f$ for which $\operatorname{Per}(f)$ is $\mathbb{R}$, we only have to verify whether $\mathbb{R}$ is $C_{1} \cup C_{2}$. For other $f$ we have to do further tests. Hereto, we now describe the construction of a continuous function $\tilde{f}$ from a given continuous function $f$.

Let $C$ denote the set $\operatorname{Per}(f)$, which, by Lemma 2 and the assumption that $\operatorname{Per}(f)$ is not $\mathbb{R}$, we can take to be $[a, b],[a,+\infty)$ or $(-\infty, b]$.

First, we collapse $C$ to $\{a\}$ if $C$ is bounded or of the form $[a,+\infty)$; and to $\{b\}$ if $C$ is of the form $(-\infty, b]$. Let us first consider the case $C=[a, b]$. Let $f_{\in C}=\{x \in \mathbb{R} \mid f(x) \in C\}, f_{<C}=\{x \in \mathbb{R} \mid f(x)<a\}$, and $f_{>C}=\{x \in \mathbb{R} \mid$ $f(x)>b\}$.

We define the continuous function $f_{1}$ on $\mathbb{R}$ as $f_{1}(x):=$

$$
\begin{cases}f(x) & \text { if } x \in f_{<C} \text { and } x<a, \\ f(x)-(b-a) & \text { if } x \in f_{>C} \text { and } x<a, \\ f(x+(b-a)) & \text { if } x \in f_{<C} \text { and } x>a \\ f(x+(b-a))-(b-a) & \text { if } x \in f_{>C} \text { and } x>a \\ a & \text { if } x \in f_{\in C} .\end{cases}
$$

This construction is illustrated in Fig. 2.

Let us next consider the case $C=[a,+\infty)$. Here, the function $f_{1}$ on $\mathbb{R}$ is defined as

$$
\begin{cases}f(x) & \text { if } x \in f_{<C} \text { and } x<a \\ a & \text { if } x \in f_{\in C} \text { and } x<a \text { or if } x \geq a .\end{cases}
$$


In the case $C=(-\infty, b], f_{1}$ is defined similarly to the previous case. Finally, we define

$$
\tilde{f}(x):=f_{1}(x+\alpha)-\alpha,
$$

where $\alpha$ is $a$ or $b$, depending on the case.

The following lemma is readily verified.

Lemma 5. Let $f: \mathbb{R} \rightarrow \mathbb{R}$ be a function. We have $f^{k}(\mathbb{R})=\operatorname{Per}(f)$ if and only if $\tilde{f}^{k}(\mathbb{R})=\{0\}$.

As mentioned in the previous section, in the area of dynamical systems, a function $\tilde{f}$ is called nilpotent if $\tilde{f}^{k}(\mathbb{R})=\{0\}$ for some integer $k$. The following lemma's show that this is a decidable property in our setting. For continuous piecewise affine functions this result was already stated (without proof) [4]. So, we extend this result to continuous semi-algebraic functions and furthermore show that the decision procedure is expressible in FO.

Lemma 6. There is an FO sentence that expresses whether a continuous semialgebraic function $f: \mathbb{R} \rightarrow \mathbb{R}$ is nilpotent.

Proof (sketch). We now describe the algorithm nilpotent(input $f$ ) to decide nilpotency of continuous semi-algebraic functions $f: \mathbb{R} \rightarrow \mathbb{R}$.

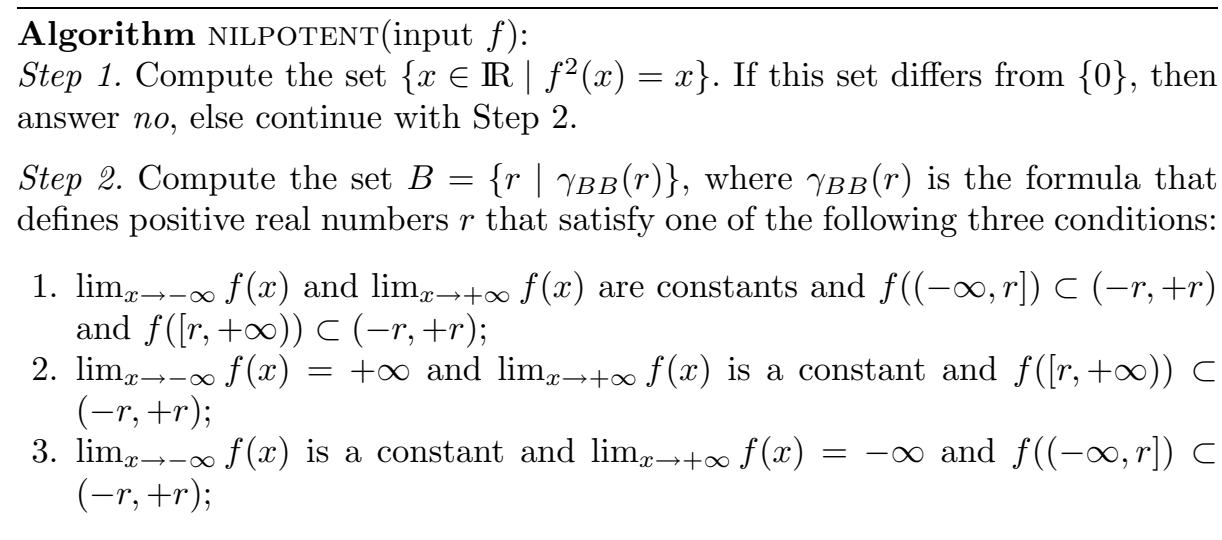

If $B$ is empty, answer no, else compute the infimum $r_{0}$ of $B$ and continue with Step 3.

Step 3. Let $g$ be the function defined as $g(x):=f(x)$ if $-r_{0}<x<r_{0}$ and $g(x):=f\left(-r_{0}\right)$ if $x \leq-r_{0}$ and $g(x):=f\left(r_{0}\right)$ if $x \geq r_{0}$. Take $r_{1}$ larger than $r_{0}$ and $\max \{|g(x)| \mid x \in \mathbb{R}\}$.

If for $g$ there exists a positive real number $\varepsilon$ such that

1. $g$ is constant 0 on $(-\varepsilon,+\varepsilon)$, or

2. $g$ is constant 0 on $(-\varepsilon, 0)$ and has a right tangent with strictly negative slope in 0 , or

3. $g$ is constant 0 on $(0,+\varepsilon)$ and has a left tangent with strictly negative slope in 0 , 
then continue with Step 4, else answer no.

Step 4. If for all $x>0, g(x)<x$ and $g^{2}(x)<x$ and for every $x<0, g(x)>x$ and $g^{2}(x)>x$ holds, then answer yes, else answer no.

We omit the proof of the correctness of the algorithm NILPOTENT.

We are now ready for the proof of Theorem 3 .

Proof of ThEOREM 3 (sketch). We describe a decision procedure TERMINATE that on input a function $f: \mathbb{R} \rightarrow \mathbb{R}$, decides whether the transitive closure of $\operatorname{graph}(f)$ terminates after a finite number of iterations.

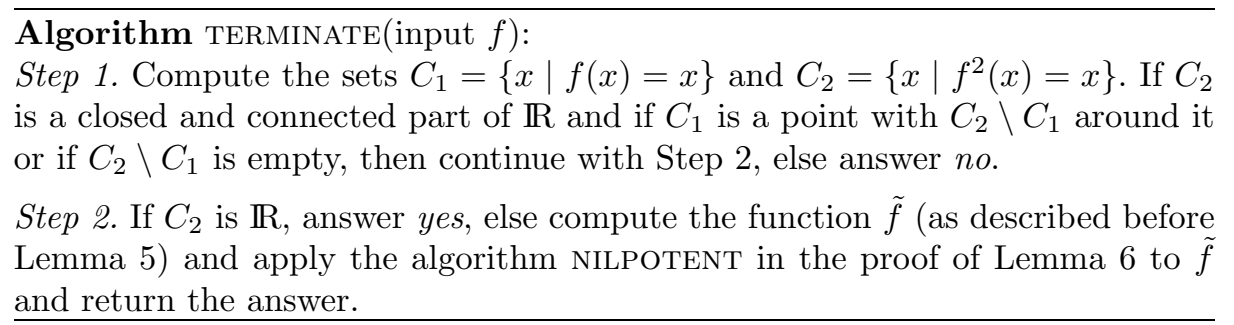

The correctness of this procedure follows from Lemmas 4, 5 and 6 . It should be clear that all ingredients can be expressed in FO.

We use the function $f_{1}$, given on the left in Fig. 1 in the Introduction, and the function $f_{2}$, given on the right in Fig. 1, to illustrate the decision procedure TERminate(input $f$ ). For $f_{1}, C_{1} \cup C_{2}$ is $\{0,1\}$, and therefore $f_{1}$ doesn't survive Step 1 and TERminate(input $f_{1}$ ) immediately returns no. For $f_{2}, C_{1} \cup C_{2}$ is $\{0\}$, and therefore we have $\tilde{f}_{2}=f_{2}$. Next, the algorithm NiLPotent is called with input $f_{2}$. For $f_{2}$, the set $B$, computed in Step 2 of the algorithm NILPOTENT, is non-empty and $r_{0}$ is 1 . So, the function $g$ in Step 3 will be $f_{2}$ again and $r_{1}$ is 1. Since $g$ is identical zero around the origin, finally the test in Step 4 decides. Here, we have that for $x>0, g(x)<x$ and also $g^{2}(x)<x$ since $x-\frac{1}{4}<x$ and $x-\frac{1}{2}<x$. For $x<0$, we have that both $g(x)$ and $g^{2}(x)$ are identical zero and thus the test succeeds also here. The output of NILPOTENT on input $f_{2}$ and therefore also the output of TERMINATE on input $f_{2}$ is yes.

For a continuous and terminating function, the periods that can appear are 1 and 2 (see Lemma 3). In dynamical systems theory, finding an upper bound on the length of the run-ups in terms of some characteristics of the function, is considered to be, even for piecewise affine functions, a difficult problem [18, 20]. Take, for instance, the terminating continuous piecewise affine function that is constant towards $-\infty$ and $+\infty$ and that has turning points $\left(0, \frac{1}{3}\right),\left(\frac{1}{3}, \frac{2}{3}-\varepsilon\right)$, $\left(\frac{4}{9}, \frac{4}{9}\right),\left(\frac{5}{9}, \frac{5}{9}\right),\left(\frac{2}{3}, \frac{1}{3}\right)$, and $\left(1, \frac{2}{3}\right)$, with $\varepsilon>0$ small. Here, it seems extremely difficult to find an upper bound on the length of the run-ups in terms of the number of line segments or of their endpoints. The best we can say in this context, is that from Theorem 3 , it follows that also the maximal run-up can be computed. 
Corollary 3. Let $f: \mathbb{R} \rightarrow \mathbb{R}$ be a continuous, terminating semi-algebraic function. The maximal run-up of a point in $\mathbb{R}$ with respect to $f$ is computable.

We end this section with a remark concerning termination of continuous functions that are defined on a connected part $I$ of $\mathbb{R}$. Let $f: I \rightarrow I$ be such a function. We define the function $\bar{f}$ to be the continuous extension of $f$ to $\mathbb{R}$ that is constant on $\mathbb{R} \backslash I$. It is readily verified that the transitive closure of $\operatorname{graph}(f)$ terminates if and only if $\bar{f}$ is terminating. We therefore have the following corollary of Theorem 3 .

Corollary 4. Let I be a connected part of $\mathbb{R}$. There is an FO expressible decision procedure that decides whether the transitive closure of the graph of a continuous semi-algebraic function $f: I \rightarrow I$ terminates.

\section{Logics with Transitive Closure Restricted to Function Graphs}

In this section, we study fragments of $\mathrm{FO}+\mathrm{TC}$ and $\mathrm{FO}+\mathrm{TCS}$ where the transitive-closure operator is restricted to work only on the graphs of continuous semialgebraic functions from $\mathbb{R}^{k}$ to $\mathbb{R}^{k}$. These languages bear some similarity with deterministic transitive-closure logics in finite model theory [8].

If $\boldsymbol{x}$ and $\boldsymbol{y}$ are $k$-dimensional real vectors and if $\psi(\boldsymbol{x}, \boldsymbol{y})$ is an FO+TCformula (respectively FO+TCS-formula), let $\gamma_{\psi}$ be the FO+TC-sentence (respectively FO+TCS-sentence) $\gamma_{\psi}^{1} \wedge \gamma_{\psi}^{2}$, where $\gamma_{\psi}^{1}$ expresses that $\psi(\boldsymbol{x}, \boldsymbol{y})$ defines the graph of a function from $\mathbb{R}^{k}$ to $\mathbb{R}^{k}$ and where $\gamma_{\psi}^{2}$ expresses that $\psi(\boldsymbol{x}, \boldsymbol{y})$ defines a continuous function graph. We can express $\gamma_{\psi}^{2}$ using the classical $\varepsilon-\delta$ definition of continuity. Therefore, it should be clear that $\gamma_{\psi}^{1}$ and $\gamma_{\psi}^{2}$ can be expressed by formulas that make direct calls to $\psi(\boldsymbol{x}, \boldsymbol{y})$. Thus, the following property is readily verified.

Proposition 1. Let $\psi(\boldsymbol{x}, \boldsymbol{y})$ be an $\mathrm{FO}+\mathrm{TC}$-formula (respectively an $\mathrm{FO}+\mathrm{TCS}$ formula). The evaluation of $\psi(\boldsymbol{x}, \boldsymbol{y})$ on an input database $A$ terminates if and only if the evaluation of $\gamma_{\psi}$ on $A$ terminates.

Definition 6. We define FO+cTC (respectively FO+cTCS) to be the fragment of $\mathrm{FO}+\mathrm{TC}$ (respectively $\mathrm{FO}+\mathrm{TCS}$ ) in which only TC-expressions of the form $\left[\mathrm{TC}_{\boldsymbol{x} ; \boldsymbol{y}} \psi(\boldsymbol{x}, \boldsymbol{y}) \wedge \gamma_{\psi}\right](\boldsymbol{s}, \boldsymbol{t})$ (respectively $\left[\mathrm{TC}_{\boldsymbol{x} ; \boldsymbol{y}} \psi(\boldsymbol{x}, \boldsymbol{y}) \wedge \gamma_{\psi} \mid \sigma\right](\boldsymbol{s}, \boldsymbol{t})$ ) are allowed to occur.

We again use superscript numbers to denote restrictions on the arities of the relations of which transitive closure can be taken.

\subsection{Deciding Termination of the Evaluation of FO + cTC $^{2}$ Queries}

Since, when $\psi(x, y)$ is $y=x+1, \gamma_{\psi}$ is true, from the proof of Theorem 2 the following negative result follows. 
Corollary 5. It is undecidable whether a given formula in $\mathrm{FO}+\mathrm{cTCS}^{2}$ terminates on a given input database.

We remark that for this undecidability it is not needed that the transitive closure of continuous functions on an unbounded domain is allowed $(f(x)=x+1$ in the proof of Theorem 2). Even when, for example, only functions from $[0,1]$ to $[0,1]$ are allowed, we have undecidability. We can see this by modifying the proof of Theorem 2 as follows. For any polynomial $p\left(x_{1}, \ldots, x_{13}\right)$, let $\sigma_{p}$ be the FO-expressible stop-condition:

$$
\begin{aligned}
& \left(\exists x_{1}\right) \cdots\left(\exists x_{13}\right)\left(\bigwedge _ { i = 1 } ^ { 1 3 } \left(\left(\exists y_{i}\right)\left(x_{i} y_{i}=1 \wedge X\left(1, y_{i}\right)\right) \vee\right.\right. \\
& \left.\left.\quad x_{i}=0 \vee x_{i}=1\right) \wedge p\left(x_{1}, \ldots, x_{13}\right)=0\right) .
\end{aligned}
$$

Since, in consecutive iterations, the function $\bar{f}$, for $f:[0,1] \rightarrow[0,1]$, with $f(x)=$ $\frac{x}{x+1}$, maps 1 to $\frac{1}{2}, \frac{1}{3}, \frac{1}{4}, \ldots$, it is then easy to see that $p\left(x_{1}, \ldots, x_{13}\right)$ having an integer solution is equivalent to

$$
\left[\mathrm{TC}_{x ; y} \operatorname{graph}(\bar{f}) \mid \sigma_{p}\right](s, t)
$$

terminating (remark again that the $\gamma_{\operatorname{graph}(\bar{f})}$ is true).

The main result of this section is the following.

Theorem 4. It is decidable whether a given formula in $\mathrm{FO}+\mathrm{cTC}^{2}$ terminates on a given input database. Moreover, this decision procedure is expressible in $\mathrm{FO}+\mathrm{cTC}^{2}$.

Proof (sketch). Given a formula $\varphi$ in $\mathrm{FO}+\mathrm{cTC}^{2}$ and an input database $A$, we can decide whether the evaluation of $\varphi$ on $A$ terminates by first evaluating the deepest FO-formulas on which a TC-operator works on $A$ and then using Theorem 3 to decide whether the computation of transitive closure halts on this set. If it does not terminate, we answer no, else we compute the result and continue recursively to less deep occurrences of TC-operators in $\varphi$. We continue this until the complete formula $\varphi$ is processed. Only if we reach the end and all intermediate termination tests returned yes, we output yes.

The expressibility of the decision procedure in FO can also be proven by induction on the structure of the formula.

\subsection{A Guarded Fragment of FO+cTC ${ }^{2}$}

The fact that termination of $\mathrm{FO}+\mathrm{cTC}^{2}$-formulas is expressible in $\mathrm{FO}+\mathrm{cTC}^{2}$, allows us to define a guarded fragment, $\mathrm{FO}+\mathrm{cTC}_{\mathcal{G}}^{2}$, of this language. Indeed, if $\psi$ is a formula in $\mathrm{FO}+\mathrm{cTC}{ }^{2}$ of the form $\left[\mathrm{TC}_{\boldsymbol{x} ; \boldsymbol{y}} \psi(\boldsymbol{x}, \boldsymbol{y})\right](\boldsymbol{s}, \boldsymbol{t})$, let $\tau_{\psi}$ be the $\mathrm{FO}+\mathrm{cTC}^{2}-$ sentence that expresses that this TC-expression terminates (obviously, $\tau_{\psi}$ also depends on the input database). We can now define the guarded fragment of $\mathrm{FO}+\mathrm{cTC}^{2}$, in which every TC-expression is accompanied by a termination guard. 
Definition 7. We define $\mathrm{FO}+\mathrm{cTC}_{\mathcal{G}}^{2}$ to be the fragment of $\mathrm{FO}+\mathrm{cTC}^{2}$ in which only TC-expressions of the form $\left[\mathrm{TC}_{\boldsymbol{x} ; \boldsymbol{y}} \psi(\boldsymbol{x}, \boldsymbol{y}) \wedge \tau_{\psi}\right](\boldsymbol{s}, \boldsymbol{t})$ are allowed.

The following property follows from the above remarks.

Proposition 2. In the language $\mathrm{FO}+\mathrm{cTC}_{\mathcal{G}}^{2}$, every query terminates on all possible input databases. Furthermore, all terminating queries of $\mathrm{FO}+\mathrm{cTC}^{2}$ are expressible in $\mathrm{FO}+\mathrm{cTC}_{\mathcal{G}}^{2}$.

\subsection{Expressiveness Results}

Even the least expressive of the transitive-closure logics is more expressive than first-order logic.

Theorem 5. The language $\mathrm{FO}+\mathrm{cTC}_{\mathcal{G}}^{2}$ is more expressive than $\mathrm{FO}$ on finite constraint databases.

Proof (sketch). Consider the following query $Q_{\text {int }}$ on 1-dimensional databases $S$ : "Is $S$ a singleton that contains a natural number?". The query $Q_{\text {int }}$ is not expressible in FO (if it would be expressible, then also the predicate nat $(x)$, expressing that $x$ is a natural number, would be in FO). The query $Q_{\text {int }}$ is expressible in $\mathrm{FO}+\mathrm{cTC}_{\mathcal{G}}^{2}$ by the sentence that says that $S$ is a singleton that contains 0 , 1 or an element $r>1$ such that $(\exists s)(\exists t)\left(\left[\mathrm{TC}_{x ; y} \psi(x, y) \wedge \gamma_{\psi} \wedge \tau_{\psi(x, y) \wedge \gamma_{\psi}}\right](s, t) \wedge\right.$ $\left.s=1 \wedge t=\frac{1}{r}\right)$, where $\psi(x, y)$ is the formula $(\exists r)(S(r) \wedge \varphi(r, x, y))$. Here, $\varphi(r, x, y)$ defines the graph of the continuous piecewise affine function that maps $x$ to

$$
y= \begin{cases}0 & \text { if } x \leq \frac{1}{r} \\ x-\frac{1}{r} & \text { if } \frac{1}{r}<x<1 \\ 1-\frac{1}{r} & \text { if } x \geq 1\end{cases}
$$

Remark that $\gamma_{\psi}$ is always true. The sentence $\tau_{\psi(x, y) \wedge \gamma_{\psi}}$ is true when the database is a singleton containing a number larger than one. The function given by $\varphi(r, x, y)$ is illustrated on the right in Fig. 1 for $r=4$. The evaluation of this transitive closure is guaranteed to end after at most $\lceil r\rceil$ iterations and this sentence indeed expresses $Q_{\text {int }}$ since $\left(1, \frac{1}{r}\right)$ belongs to the result of the transitive closure if and only if $r>1$ is a natural number.

\section{Concluding Remarks}

We conclude with a number of remarks. One of our initial motivations to look at termination of query evaluation in transitive closure logics was to study the expressive power of $\mathrm{FO}+\mathrm{TC}$ compared to that of FO+TCS. As mentioned in the Introduction, the latter language is computationally complete on linear constraint databases. It is not clear whether FO+TC is also complete. In general, we have no way to separate these languages. But if we restrict ourselves to their fragments $\mathrm{FO}+\mathrm{cTC}^{2}$ and $\mathrm{FO}+\mathrm{cTCS}^{2}$, the fact that for the former termination is 
decidable, whereas it is not for the latter, might give the impression that at least these fragments can be separated. But this is not the case, since equivalence of formulas in these languages is undecidable. In fact, the expressions used in the proof of Theorem 2, are expressible in FO+TC (they do not even use an input database).

A last remark concerns the validity of the results in Section 4 for more general settings. Lemmas 1-5 are also valid for arbitrary real closed fields $R$. One could ask whether the same is true for Lemma 6 . However, the proof of the correctness of the FO-sentence which decides global convergence in Step 4 [3], relies on the Bolzano-Weierstrass theorem, which is known not to be valid for arbitrary real closed fields [5]. Furthermore, we can even prove that no FO-sentence exists that decides termination of semi-algebraic functions $f: R \rightarrow R$ for arbitrary real closed fields $R$.

Acknowledgments. We thank the reviewers for a number of suggestions to improve the presentation of our results.

\section{References}

1. Ll. Alsedà, J. Llibre, and M. Misiurewicz. Combinatorial Dynamics and Entropy in Dimension One, volume 5 of Advances Series in Nonlinear Dynamics. World Scientific, 1993.

2. M. Benedikt, M. Grohe, L. Libkin, and L. Segoufin. Reachability and connectivity queries in constraint databases. In Proceedings of the 19th ACM SIGMODSIGACT-SIGART Symposium on Principles of Database Systems (PODS'00), pages 104-115. ACM, 2000.

3. V.D. Blondel, O. Bournez, P. Koiran, C.H. Papadimitriou, and J.N. Tsitsiklis. Deciding stability and mortality of piecewise affine dynamical systems. Theoretical Computer Science, 255(1-2):687-696, 2001.

4. V.D. Blondel, O. Bournez, P. Koiran, and J.N. Tsitsiklis. The stability of saturated linear dynamical systems is undecidable. Journal of Computer and System Sciences, 62(3):442-462, 2001.

5. J. Bochnak, M. Coste, and M.-F. Roy. Real Algebraic Geometry, volume 36 of Ergebenisse der Mathematik und ihrer Grenzgebiete. Folge 3. Springer-Verlag, 1998.

6. G.E. Collins. Quantifier elimination for real closed fields by cylindrical algebraic decomposition. In Automata Theory and Formal Languages, volume 33 of Lecture Notes in Computer Science, pages 134-183. Springer-Verlag, 1975.

7. M. Davis, Y. Matijasevič, and J. Robinson. Hilbert's Tenth Problem. Diophantine equations: positive aspects of a negative solution. In Mathematical Developments Arising from Hilbert Problems, volume 28, pages 323-378. American Mathematical Society, 1976.

8. H.-D. Ebbinghaus and J. Flum. Finite Model Theory. Springer-Verlag, 1995.

9. F. Geerts. Linear approximation of semi-algebraic spatial databases using transitive closure logic, in arbitrary dimension. In G. Ghelli and G. Grahne, editors, Proceedings of the 8th International Workshop on Database Programming Languages (DBPL'01), volume 2397 of Lecture Notes in Computer Science, pages 182-197. Springer-Verlag, 2002. 
10. F. Geerts and B. Kuijpers. Linear approximation of planar spatial databases using transitive-closure logic. In Proceedings of the 19th ACM SIGMOD-SIGACTSIGART Symposium on Principles of Database Systems (PODS'O0), pages 126135. ACM, 2000.

11. S. Grumbach and G. Kuper. Tractable recursion over geometric data. In G. Smolka, editor, Proceedings of Principles and Practice of Constraint Programming (CP'g7), volume 1330 of Lecture Notes in Computer Science, pages 450-462. SpringerVerlag, 1997.

12. P.C. Kanellakis, G.M. Kuper, and P.Z. Revesz. Constraint query languages. Journal of Computer and System Science, 51(1):26-52, 1995. A preliminary report appeared in the Proceedings 9th ACM Symposium on Principles of Database Systems (PODS'90).

13. P. Koiran, M. Cosnard, and M. Garzon. Computability with low-dimensional dynamical systems. Theoretical Computer Science, 132:113-128, 1994.

14. S. Kreutzer. Fixed-point query languages for linear constraint databases. In Proceedings of the 19th ACM SIGMOD-SIGACT-SIGART Symposium on Principles of Database Systems (PODS'00), pages 116-125. ACM, 2000.

15. S. Kreutzer. Operational semantics for fixed-point logics on constraint databases. In R. Nieuwenhuis and A. Voronkov, editors, Proceedings of the 8th International Conference on Logic for Programming, Artificial Intelligence, and Reasoning (LPAR'01), volume 2250 of Lecture Notes in Computer Science, pages 470-484. Springer-Verlag, 2001.

16. S. Kreutzer. Query languages for constraint databases: First-order logic, fixedpoints, and convex hulls. In J. Van den Bussche and V. Vianu, editors, Proceedings of 8th International Conference on Database Theory (ICDT'01), volume 1973 of Lecture Notes in Computer Science, pages 248-262. Springer-Verlag, 2001.

17. G.M. Kuper, J. Paredaens, and L. Libkin. Constraint databases. Springer-Verlag, 1999.

18. J. Llibre and C. Preston. Personal communication. 2002.

19. Y. Matiyasevich. Hilbert's Tenth Problem. The MIT Press, 1993.

20. C. Preston. Iterates of Maps on an Interval, volume 999 of Lecture Notes in Mathematics. Springer-Verlag, 1983.

21. A. Tarski. A Decision Method for Elementary Algebra and Geometry. University of California Press, 1951. 